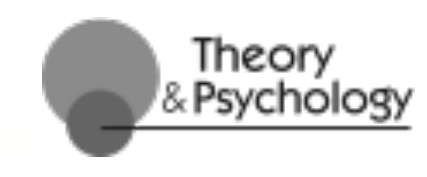

Article

\title{
The motivational role of affect in an ecological model
}

\author{
Rami Gabriel \\ Corresponding author: \\ Rami Gabriel, Ph.D., Columbia College Chicago, 600 S. Michigan Avenue, Chicago, IL 60605-1996, USA. Email: rgabriel@colum.edu
}

\begin{abstract}
Drawing from empirical literature on ecological psychology, affective neuroscience, and philosophy of mind, this article describes a model of affect-as-motivation in the intentional bond between organism and environment. An epistemological justification for the motivating role of emotions is provided through articulating the perceptual context of emotions as embodied, situated, and functional, and positing perceptual salience as a biasing signal in an affordance competition model. The motivational role of affect is pragmatically integrated into discussions of action selection in the neurosciences.
\end{abstract}

\section{Keywords}

affect, affordances, ecological psychology, motivation, philosophy of psychology

The study of emotions in recent decades has enabled researchers to rethink the study of the mind (Damasio, 2018; Davidson et al., 2009; de Waal, 2001; Panksepp, 1998; Pessoa, 2013; Phelps, 2006; Prinz, 2004). Across roughly the same period, there has been a surge in creative research and theory in the field of ecological psychology (Chemero, 2009; Heft, 2007; Reed, 1996; Rietveld \& Kiverstein, 2014; Turvey, 1992; Withagen \& Chemero, 2009). Both affective neuroscience and ecological psychology arise from reflection upon the insights, and shortcomings, of prevalent methodologies in the cognitive sciences, social psychology, and machine learning. These shifts in focus, from cognitive to affective aspects of mind and the renewed emphasis on bodily-perceptual explanations of behavior rather than computational mechanics, can work synergistically. In this article, I argue that clarify- ing the motivational function of affective sentience in perceptual experience and various forms of navigation as intentions-in-action will clarify our understanding of bias, salience, and attention as studied in contemporary neuroscience. ${ }^{1}$

A useful definition of affect will require coming to terms with the nature of biological intentionality. Specifically, the motivational function of emotion must be located in a neoAristotelian notion of animal striving. Spinoza's (1677/2001, Ethics, Part III, prop. 6) conatus 
captured this goal-oriented tendency of living creatures, but more recent acknowledgment of the psychological and neurobiological shape of such a system of incentive salience and "wanting" is

now available. ${ }^{2}$ The basic conative intentional role of affect is to provide the animal with motivated direction in its exploration of the world (Damasio, 2018). The SEEKING system is a neuroanatomical description of this motivational thrust, "a goad without a goal,"3 responsible for: (a) general behavioral activation, (b) the felt experience of wanting that mediates incentive valence, (c) persistence of behavior, (d) shifting between behavioral sets, (e) simple approach behavior, and (f) reward prediction error (Panksepp, 1998). This SEEKING system is in continuous oper- ation and is characterized by an intense, enthused curiosity about the world; for example, humans in the throes of these mesolimbic and mesocortical dopamine pathways report a "can-do" feeling as an enhanced sense of themselves as effective agents (Panksepp, 1998). As I describe below, motivational significance and incentive value of cues and expected outcomes appears to be encoded in the orbital region of the prefrontal cortex (PFC; Schoenbaum et al., 1998).

Basic affect conceived as motivation clarifies the phenomenal experience of value. Affect functions as a self-regulatory motivational process of informed awareness of one's needs, wants, intentions, preferences, values, priorities, and goals such that the greater the ecological significance, the more likely a phenomenon will be attended to (Gibson, 1979; Shaw \& KinsellaShaw, 2007). Affect thus plays a crucial role in shaping act-planning via elaborations of learned associative relations between mind, body, and world that are manifested as intentions-in-action through salience and attentional effects.

My purpose in this paper is to justify this motivational role of emotions by describing recent expansions to ecological psychology that demonstrate that emotions are embodied, situated, and functional, and, in the final sections, to connect perceptual salience to an affordance competition model from the neurosciences.

\section{Ecological psychology and the extended evolutionary synthesis}

Ecological psychology has enlarged the range of behaviors to which we can emphasize bodyworld loops of psychological causality. It embraces the dynamic nature of locomotion by insisting on the fundamental properties of change and the intrinsic and relational properties of organism-environment interaction (Gibson, 1967). Recent work has broadened such an approach to the situatedness of behaviors elicited in dyadic relations (McCall \& Singer, 2015), and to how behavior is elicited by features of architectural space (Robinson \& Pallasmaa, 2017). Direct perception as well as niche construction allow us to understand how human (and broadly mammalian) behaviors, from locomotion to affordances garnered during social interaction, arise from perception-action processes mediated by emotions. This section details progress in the study of affordances concerning social interaction and the built environment in order to secure space for the role of affect as an interface between perception and motivation in the next section.

One perceives to act, and acts to perceive; therefore, the value and meaning of a given percept or object in the world consist in what it affords the perceiving agent. The properties of the environment to which an animal is directed are perceptual invariants, such as the kinematic specification of dynamics, which grant information concerning causal factors of events 
(Michaels et al., 2001; Runeson, 1988). Perception-action systems produce dispositions for the animal relative to the environment; specifically, affordances are dispositions to act gleaned from perception. Effectivities are the animal's bodily dispositions in relation to the perceived surface (Withagen \& Michaels, 2005), or that which seizes and actualizes affordances (Shaw, 2003). An actor chooses affordances to be realized, a corresponding mode of action, and the appropriate laws of control by which to regulate the action (Warren, 1988). Global action modes consist of ethological behavior maps nested in subsidiary order task-specific groupings of musculature organization (Graziano, 2016; Iberall \& McCulloch, 1969). An organism's exploration of the environment includes prospective control, that is, modifying its relation to the environment to perceive particular affordances in the context of goals to be realized (Pezzulo \& Cisek, 2016; Turvey, 1992).

In addition to physical ecology, our niche is sociocultural: people afford "the whole spectrum of social significance" (Gibson, 1979, pp. 127-128). That is to say, the social- ity of homo sapiens is such that individuals need other individuals, from neonatal care relationships to coalitionbuilding, to apprentice learning of sophisticated skills. Interaction between people takes place in a sociocultural niche, wherein communication and social position provide signals that may quickly be read as affordances (Laland et al., 2010; Warren, 2006). Social affiliation is crucial for evolutionary success in humans, and therefore perception of possibilities for social interaction may be an evolutionary feature of mind (Runeson \& Frykholm, 1983; Van Acker \& Valenti, 1989). The social niche is a background condition for mammalian ecological psychology, it is a habitat fashioned by the animals who occupy it (Drake, 2003; Heft, 2007). Fundamental social action modes and motives include: care for offspring, forming and maintaining social alliances, seeking status, self-protection, attracting mates, and retaining mates (Neuberg et al., 2010). Accordingly, imitation and emulation are inborn forms of social learning (Fiebich, 2014). The social element is psychologically constitutive in child socialization (Cole, 2006; Cole et al., 1996; Ellis, 1997; Reed, 1996; Rogoff, 2003), and joint attention (Tomasello, 2000). Individuals are sensitive to other individuals in their environment, their action capabilities, and the affordances of social synergy in interpersonally co-ordinated action (Marsh, 2015).

Expressed emotions are thus a form of social affordance. ${ }^{4}$ The social function of emotional display as a social signal to change the behavior of other organisms is part of the mutual concerns that develop in relationships (Frijda, 1986). As such, they can be under- stood as skillful engagements with the world for the purposes of influencing the percep- tion and behavior of conspecifics (Griffiths \& Scarantino, 2009). For example, Fischer and Manstead (2015) describe the affiliative and distancing functions of positive and negative emotions at the interpersonal and group levels, arguing that the primary social function of emotions is social distancing or affiliation. Nonverbal expressions of others thus provide a signal, a social appraisal that has an effect on one's own emotional state (Manstead \& Fischer, 2001). Calibration of one's body image, and thus its effectivities, is highly dependent on elements like body percept, body concept, and body affect gleaned from interaction with others and the environment (Cacioppo et al., 2018; Lo Presti, 2020). Entrainment of the body and emotional reactions occurs during conversation as a form of participatory co-ordinated social sense making (de Jaegher \& di Paolo, 2007; Wilson \& Wilson, 2005). 
The social function of emotions consists of:

First, positive group-based emotions ... promot[ing] group cohesiveness, ingroup identification, and ingroup cooperation. Second, ingroup bonds can also be strengthened by emotions that serve a distancing function when these are expressed toward deviant group members ... Third, certain negative group-based affiliative emotions, such as guilt and shame, may strengthen bonds within groups because they are collectively experienced as aversive and/ or threatening to the group's social identity. ... Fourth, group bonds can also be strengthened by differentiating one's group from other groups and/or challenging the social hierarchy by expressing anger, hate, or contempt towards outgroups that are seen as threatening the ingroup's interests or values. At the same time, positive emotions, such as admiration for another group, may also help to maintain social hierarchies. Finally, positive intergroup relations can be promoted by group-based anger. (Fischer \& Manstead, 2015, p. 14)

More specifically, there are social affordances that reflect one's position in the social hierarchy; these include gaze, head angle, and smiling during listening (Ashenfelter et al., 2009; Cashdan, 1998; Ellyson et al., 1980; Mignault \& Chaudhuri, 2003). Responses to such proxemic bodily cues are approach and avoid behaviors correlated with autonomic responses (Dovidio et al., 1988; McCall et al., 2009). Nonverbal communication is expressive behavior displayed in nonlinguistic creatures:

signals such as grooming, grunts, food-begging gestures, nursing pokes, and ground-slaps, and various other "intention movements" and "attention getters," form unlearned shared repertoires of expressive vehicles. . . bearers of specific informative signification. (Bar-On, 2017, p. 306)

Just as we store memory in external devices and augment our cognition with technology, affect can be scaffolded beyond the body when we curate an environment of objects in urban and interior design to elicit affective states (Krueger \& Szanto, 2016). Humans build an affective niche of their environment; from trust in material and relational elements of built structures to habits, tools, collective norms, and informational prostheses (Colombetti \& Krueger, 2015). The built environment itself, in this context through its industrial design, elicits action possibilities (Heft, 2010; Ju \& Takayama, 2009; Norman, 2002). Landscape affordances are arenas for idiosyncratic and intra-psychic value-rich environmental experience (Heft, 2010). Take, for example, how the design of a public transportation train station communicates from the mind of the designer to the mind of the user through perceptual features like changes in texture, visual sight, and sound. The phenomenological aspect of this Gestalt solicitation to act given by the landscape affordances is functional (Dreyfus \& Kelly, 2007).

Organisms actively construct and modify environmental conditions in nonrandom ways that bias their own survival and benefit the lifeways of their offspring (Lewontin, 1984). In effect, locomotive animals forge a set of affordances, which serve as relational resources to ease the cognitive load of movement through the world; this can range from nest construction to the construction of dams and underground burrows. The Extended Evolutionary Synthesis (EES) states that "developmental processes, operating through developmental bias, inclusive inheritance and niche construction, share responsibility for the direction and rate of evolution, the origin of character variation and organism-environment complementarity" (Laland et al., 
2015 , p. 1). For example, parents modify ecology to provide safety and shelter for their young (Badyaev \& Uller, 2009). Such considerations entail an ecological-developmental perspective on the evolved nature of embodied mind; a niche construction perspective provides a level of analysis between natural selection and domestication (Laland et al., 2015, 2017). In conjunction with eco- logical psychology, the EES makes possible a conceptual platform to discuss a wide range of instances when thoughts and achievements are set into material culture, thus problematizing the distinction between subjective and objective features of the land- scape (Heras-Escribano \& de Pinedo-García, 2018). Indeed, social institutions serve as an implicit background for interpersonal interactions; they are producers and maintainers of affordances (Kono, 2009). Cultural affordances, like written language and historical ruins, enable developmental acculturation (Ramstead et al., 2016). ${ }^{5}$ These epistemic affordances from body, object, and place create possibilities for reflection.

In sum, recent expansions of the application of direct perception emphasize how behavior is a "regulatory" and functional ecological mode of resource usage (Reed, 1986, 1996). My aim here is to demonstrate how the notion of affordances has been extended from the traditional sensorimotor varieties to intentional shared attention and institutional affordances, like organizational structures of social interaction (Fiebich, 2014). Intentionality-in-action as perception is thus an embodiment of the relation between interoceptive needs of the homeokinetic system and external resources (Damasio, 2018; Gibson, 1979; Iberall, 1995). From niche construction to ecological approaches, the situatedness of behavior clarifies how action is determined through the relation between organism and environment.

\section{Cultural affordances and the role of affect}

In this section, I discuss cultural affordances and introduce the role of affect in an expanded ecological frame. Culture and context shape human behavior by integrating embodied, cognitive, and affective substrates of action into lived experience (Ramstead et al., 2016). Cultural norms that elicit behaviors in particular situations - for example, the proper distance to stand from another person, or bodily orientation - require learning for their entrainment and implicitly communicate attitudes and motivations (McCall \& Singer, 2015). While direct perception seems best suited for valid and reliable law-based information, learned elements from sociocultural entrainment, like conventions, can become affordances (Golonka, 2015).

Sociocultural affordances that require learning, like appropriate eye contact or circumscribing circles of intimacy, become bodily dispositions in the perceiving agent. The act of detecting information that specifies a given affordance must be built atop homeo- static needs and include the sculpting of perceptual abilities within the individual's built social, cultural, and material environmental niche. Neoteny is the crucial period for enculturation in primate infants, wherein the infant-caregiver relationship shapes elements of affective response and social intelligence through eye contact, smiling, and motherese (Narvaez et al., 2012). De Jaegher and di Paolo (2007) suggest that the interactional domain may be an autonomous level of emergent properties. Whereas such law- like information as environmental invariants plays a role in the continuous control of behavior, we need to understand how conventions that arise from culture and accident play a role in creating biases in the process of action selection. Part of the project of expanding ecological psychology into sociocultural and landscape domains, including conspecifics as 
opportunities for affordances, leaves some unresolved issues concerning how to best incorporate normativity and cultural differences, for example in the case of manners (Lo Presti, 2020).

To solve this issue of conventions, Rietveld and Kiverstein (2014) draw on the work of Viennese philosopher Ludwig Wittgenstein to describe forms of life as stable habitual patterns that become normative behavior as customs in a community. Information is established by grounding in rules of reference that specify what symbols refer to, or denote, and by situating, which requires rules of usage that specify what the symbol means in context (Shaw, 2003). The affordances the environment offers are then dependent on the abilities of the creature within the set of conventions and cultural resources available. Informed awareness thus acts as a constraint on action rules (Shaw, 2003). The social relationships involved in the conventions of teaching and learning display a form of situated normativity, wherein skill acquisition is a form of educating the attention (Sterelny, 2012). Bodily cues like posture, sustained eye contact, and prosody are perceived as social affordances that suggest the appropriate manner for directing attention. Engaging adequately with an affordance is thus itself a type of skill wherein the demand character or solicitation of an affordance becomes relevant to the creature's concern. The solicitation displayed by the animal forms part of the "action readiness" and bodily potentiation garnered by the built environment (Frijda, 2007; Rietveld, 2012; Rietveld et al., 2013).

The expanded notion of ecological psychology presented thus far allows us to invigorate an understanding of the functional role of affect-as-motivation within developmentally inscribed goads for behavior at multiple levels of perception. The existential evaluative sense of affect-asmotivation is crucial for grounding a broader model of the sociocultural niche and learned ontologies in felt experience. Affect as salience takes the form of goads that covary with homeostatic/homeokinetic needs and lead to ethological action modes, such as further information-seeking behaviors. Manipulating and regulating goals in the context of an animal's interaction with its niche leads to global effects upon behavior and action selection.

Anatomically, the felt sensation and behavioral effects of this affect-as-motivation towards environmental resource goals is facilitated by Deep Brain Stimulation (DBS) of the transdiencephalic medial forebrain (Panksepp \& Biven, 2012). The nucleus accumbens (NA) in particular serves as a motivational gateway because motor control and emotional signals, especially from the ventral striatum, engender a stimulation that comes to be manifested as enthusiasm and future-planning (Panksepp, 1998). This energized forward locomotion is the paradigmatic behavioral aspect of SEEKING and is observable in diverse exploratory foraging and investigatory activities. The ability to respond to affordances mediated by this system is adversely affected by DBS of the NA, as well as frontostriatal pathways (de Haan et al., 2013; Figee et al., 2013). Researchers observed that a hospitalized DBS patient's experience of the world changes in response to DBS to the areas in question; first, there is a modification of patients' field of affordances on a set of dimensions that includes broadness of scope and temporal horizon. Second, patients' social interactions are modified, implying a crucial role for affect and social affordance in patients' experience of moods and feelings in response to social interaction, as well as their own subsequent experience of such deficiencies. For example, patients commonly report that when DBS is effective in stimulating the NA, there is a lessening of the experience of tension and anxiety paired with an increase in self- esteem, which results in more speaking out, expressive behaviors, and assertiveness; these changes make patients feel 
more like interacting with others (de Haan et al., 2013). de Haan et al. (2013) claim DBS treatment transforms the field of affordances insofar as patients' interest in possibilities for action broadens; this is reflected in the increased width of their field of affordances and how the patients orient themselves on a larger time scale of increased depth. Their detection of relevance in stimuli becomes more in line with the totality of their concerns, such that affordances come to be differentiated in terms of relevance (p. 8). Not surprisingly, the patients' existential sense of evaluative stance-taking, which is comprised of second order cognitive reflections upon allostatic short and long-term needs, is compromised (p. 8).

The modifications caused by such anatomical interventions illustrate the ecological notion that we perceive the world relative to what we can do and what we are motivated to do. Intentions are the species that emerge from the competition among perception and action processes to utilize affordances adaptively (Frijda, 2007). A crucial feature of the changes in the DBS patients' relation to the world is in the relevance, that is, "height," or salience, of perceived properties. Below, I take up this connection between direct perception and affect as salience in prefrontal action selection.

\section{Affect-as-motivation in an affordance competition model}

One viable approach to connect the direct perception framework with prefrontal net- works for decision making is in the conjunction of affect and affordances explained in Cisek's (2007) affordance competition model. I operationalize the motivational function of affect as bias, salience, and attentional effects in line with the notion of SEEKING and wanting provided above. In the affordance competition model, behavior is the product of a competition between the parallel processing of actions in the dorsal visual system of the frontoparietal cortex. The influences that bias the selection of affordances are salience and attention, as well as reward and expectation variables from prefrontal regions and the basal ganglia, which come to bear upon action selection in executive and motor areas (McGinty et al., 2016). Cisek (2007) argues that the brain processes for both how to do a set of possible actions and selection of the particular appropriate action occur simultaneously in a hierarchical affordance competition with the dorsal stream specifying parameters of potential actions and the ventral stream providing further information towards selection. The dorsal stream of the visual system diverges into a set of substreams for action modes and way-finding in an affordance landscape. This approach suggests the brain is a feedback control system whose goal is to guide interaction with the world, which accords with the homeokinetic ecological interpretation rather than the sense-think-act representational models of cognitive science (Pezzulo \& Cisek, 2016). Cisek and Kalaska (2010) claim "the classical assumption of a unified and stable internal representation (an internal replica of the external world) does not appear to be well sup- ported by the divergence of the visual system and the widespread influence of attentional and contextual modulation" (p. 273). While neuroscience largely adapts technical terms that connote internal processing of representations, the findings of the field do not necessarily imply the computational theory of mind. First of all, this is because there are alter- native interpretations of intermediate entities, including embodied representations and Pushmi-pullyu Representations (PPRs) (Hufendiek, 2018; Millikan, 1996; Pezzulo, 2011). Second, the explanatory elements employed in neuroscience are at a different level of interpretation than those of cognitive psychology (Fodor, 1998; Gabriel, 2012). ${ }^{6}$ That perception and behavior are directly guided by dynamic perceptual interaction with the world 
does not entail that the brain is a direct, representational system. The brain contains a vast amount of learned associations to allow for appropriate responses to a variety of ethological situations, Cisek's affordance competition model can help us account for this neural process in ecological terms. That one or more pathways are put into use rather than others is a description at the level of a physical system that functions via connections, assemblies, and neuromodulation; there is no need to posit a functional parallel between the act of perception and the action of neural matter. 7

A related research endeavor explains how the lateral intraparietal area hosts an atten- tional mechanism that represents a salience map of the visual field (Gottlieb et al., 1998; Kusunoki et al., 2000). Salience in this region is said to reflect affective coloring of action affordance options manifested in intended saccadic eye movements as attentional attractors. Further downstream in the dorsal system there is evidence that attention modulates which information is more important and which should be suppressed (Desimone \& Duncan, 1995). This biasing factor modulates activity in frontal neurons reflecting the expected utility as well as subjective desirability of a range of variables in parietal neurons (Dorris \& Glimcher, 2004). Biasing inputs originate in the basal ganglia or the PFC, specifically the dorsolateral prefrontal cortex (DLPFC; Cisek, 2007). ${ }^{8}$ Subjective value signals that help determine action specification originate in the orbitofrontal cortex (OFC; Cisek \& Kalaska, 2010; Wallis, 2007). The affordance competition hypothesis suggests choices emerge through the activity of probability density functions of potential movements within the same neural populations in the assemblies that control the execution of action.

The structure of the OFC is similar across primates and appears to integrate sensory and reward information (Wallis, 2007). Neural signals concerning past and possible rewards reflect affective processing in the OFC. We can surmise this function through observation of OFC brain damage patients who seem unable to make basic decisions, which may devolve into issues of inability to reverse or extinguish previous learning (Damasio, 1994; Rolls et al., 1994). Lesions in the OFC cause difficulty in completing tasks that require making choices, suggesting an inability of a cue to access the incentive value of a learned association (Gallagher et al., 1999). Studies suggest the OFC encodes the value of a choice outcome and then passes the information to the DLPFC, which subsequently uses the information to control a range of behaviors (Wallis, 2007). The somatic marker hypothesis (Bechara et al., 1994) suggests that the OFC uses autonomic signals to guide decision making by storing associations between patterns of environ- mental and somatic input that bias decision making (Cisek \& Kalaska, 2010). The biasing functions of the OFC are partly responsible for action selection in an affordance competition model; I argue this provides an illustration of affect-as-motivation.

Anatomically, the motivational function of value resides in OFC frontostriatal dopaminergic projections from the basal ganglia (Schultz et al., 2000). The OFC receives connections from all sensory modalities and extensive limbic connections, in addition to the autonomic nervous system (ANS) and the periaqueductal grey, though it has weak motor connections (Wallis, 2007). Biasing signals therein take the form of motivational affective nudges wherein value in the OFC is an instantiation of computationally efficient preference transitivity adapted over a range of decisions (Platt \& Padoa-Chioppa, 2009). Such a conative-doxastic loop may assign a cardinal measure of values to situation-types and action modes, or ethological action maps (Graziano, 
2016; Iberall, 1995; Pollock, 2001). Economic choices may be partly based on such value signals in the OFC, wherein the three elements of the motivational value of reward are quality, quantity, and probability of reward. Criterion setting and attentional dynamics are also crucial factors in decision making (Crapse et al., 2018).

The dopaminergic activity in the striatum discussed above as part of the SEEKING system is involved in approach behaviors, reward-related learning, and addiction (Fibiger \& Phillips, 1986; Wise \& Rompré, 1989). In macaques, the OFC shows three forms of activity: response to reward prediction instructions, activity during expectation or immediately before reward, and response following reward (Schultz et al., 2000). The goal- directed actions implicated highlight the situatedness of behavior insofar as action selection is based on learned associations of contingency between choice and outcome (Dickinson \& Balleine, 1994). The OFC may integrate multiple sources regarding reward outcome to derive a value signal; specifically, it calculates how rewarding a reward is and this value signal can then be used by the lateral prefrontal cortex in planning and organizing behaviors as well as by the medial prefrontal cortex to evaluate overall action, success, and effort required (Wallis, 2007). Reward can be separated into two felt signals of affect-as-motivation: hedonic value, that is, how much we like something, and incentive value, that is, how much we want something, or affective salience.

In sum, the existential evaluative sense operationalized in the variable of "height" in de Haan et al. (2013), the felt goad of the SEEKING system (Alcaro \& Panksepp, 2011), and the somatic marker hypothesis (Damasio, 1996) as forms of affect-as-motivation map onto the affective signals generated in the basal ganglia and DLPFC, and are syn- thesized in the OFC as part of the affordance competition model.

\section{Reward and motivation}

In this section, I expand notions of reward and affect-as-motivation into the landscape of affordances and decision making. Response to reward can be attuned by secondary rein- forcers, that is, developmentally acquired learned associations that coincide with secondary level affective processes such as learning and enculturation. Though it is a crude mechanism, conditioning remains the best contender for how we attach value to beliefs and perceptual states in the absence of innate concepts (Fodor \& Pylyshyn, 1981; Pollock, 2001; Turvey et al., 1981). We know that crossmodal associations are made in the OFC of rodents (Lipton et al., 1999), and there is evidence that multiple drafts of the conditioned stimulus-unconditioned stimulus (CSUS) relation are invoked in the OFC that may provide the basis for initial learning. Conditioned stimuli can themselves acquire incentive value and serve as the US in support of new learning as secondary reinforcement (Gallagher et al., 1999). Learned secondary reinforcers conceivably include social and cultural affordances of the built environment, including conventions and landscape affordances of the evolutionary extended niche.

How might affect as a marking of associatively encoded valence in situated contexts play a role in decisions? Decision making essentially has three parameters: the expected payoff, the costs in time and energy, and the probability of success (Kahneman \& Tversky, 2000). In this case, the medial PFC is a good candidate for the encoding of an amalgamated abstract value signal, whereas the OFC may encode the value of expected or potential goals and outcomes (Wallis, 
2007). Notably, lesions of OFC in monkeys have been found to disrupt the functioning of secondary reinforcers (Pears et al., 2003). The value representation in the medial PFC may be encoded either as learning of association (e.g., a hedonic representation - the experience of "liking") or as an incentive repre- sentation (the experience of "wanting"; Wallis, 2007). These processes reflect different aspects of the pleasure system, with "wanting" more closely aligned with the SEEKING system as a form of conative motivation (Berridge \& Kringelbach, 2013). The SEEKING system could be considered part of a neural clearinghouse where potential actions and their associated affective states compete and modify each other toward unfolding action plans (Watt, 2017). Particular allostatic needs may be filtered through the SEEKING system depending upon temporal factors like immediacy of need, as well as longer term mood envelopes and the situational context of the perceived landscape of affordances.

While I have been emphasizing internal goals (allostasis) and motivational states, affect is also an encoding of exogenous needs. The competition between internal and exogenous needs is represented in subcortical enervations of frontoparietal regions and manifests itself in attentional effects (Pessoa, 2013), and on affordance competition selection (Cisek, 2007). For example, anxious individuals tend to allocate excess attention to threat, and such alterations in amygdalahippocampal emotional functionality tend to persist for more than 2 hours after the intense social stress (Okon-Singer et al., 2015). This accords with research on how developmental enculturation shapes forms of coping and other emotional lifeways (Narvaez et al., 2012). One of the ways in which emotional experience, like threat distractors, transforms an individual's behaviors and dispositions to act is that it creates a filtering inefficiency that hijacks endogenous attention from other types of top-down control (Okon-Singer et al., 2015). Social information, like the social affordances discussed above, may result in changes of subjective value signals from the OFC (Chang, 2017). This has been observed in reward value gain modulation of the egocentric-encoding neurons in the OFC and anterior cingulate cortex (ACC; Chang et al., 2013). The value tuning of individual neurons in the OFC seem to be systematically modulated by gain as a function of the position of the perceived con- specific in the social hierarchy (Azzi et al., 2012).

Motivation thus appears to alter many aspects of sensory processing; not only by modifying OFC activation, but also in shifting the timing of response (Pessoa, 2013). The view that arises from such empirical work suggests that affect-as-motivation, as salience or action selection bias, reduces response conflict or task-switch costs, via selective effects on attention and efficiency in the use of long-term memory. Motivation may enhance task processing so as to increase the likelihood of attaining a reward; anticipation of reward enhances stimulus processing and reduces interference from conflicting information streams (Padmala et al., 2011).

If the brain is conceived of as an interactive, heterarchical structure of distributed control, the distinction between emotion and cognition then devolves into a distinction between modes of modulating general psychological processes, such as attention (Pessoa, 2018). A heterarchical vision of brain function would allow for increased attentiveness to informational variables specifying the desired object or event (i.e., sensitivity to con- text), such that direct perception signals have a situated, continuous modulatory effect on associational processes (Zipoli Caiani \& Ferretti, 2017). Specifically, integrated combinatorial circuits responding to the landscape of affordances would afford interaction between appetitive, aversive, and contextual elements of 
both cortical and subcortical structures (Pessoa, 2018). While further complicating an already complicated topic, this interpretation aids in our understanding of pronounced experimental effects like mood- congruency (Harlé et al., 2010), as well as modulation of sensitivity to reward, attentional avoidance, and emotional distraction (Okon-Singer et al., 2015). For example, it clarifies how the midcingulate cortex can act as a hub for information about pain, threat, punishment, and negative feedback while biasing signals in regions involved in the expression of fear, anxiety, and goal-directed behaviors, such as selective attention (Shackman et al., 2011).

As energized by subcortical SEEKING or wanting circuits, motivation has some of its effects via recruiting attentional circuits to increase sensory sensitivity. That is to say, motivation circuits are embedded into cognition and perception in such a manner as to manifest themselves in the shifting of resources, in modifying how an individual perceptually navigates the world. Affect as a form of motivation thus takes on a quasiglobal role in a functionally integrated model of biological intentionality. I have argued that this role is observed in bias and attentional salience of the OFC within an affordance competition model of direct perception centered in the dorsal visual pathway with inputs to PFC action selection from parietal and basal ganglia sites. Affect may thus mediate between goal states by modulating domain general aspects like attention and salience in decision making and affordance selection.

\section{Discussion}

I have argued that affect-as-motivation is an integral factor in how the brain as a heterarchical affordance competition feedback control system responds to the landscape of affordances. Primary conative affect (i.e., SEEKING) is enhanced by secondary-level conditioning (learning and enculturation) and tertiary-level cortical and cognitive elements during development to provide a functional biasing signal for the representation of values in action selection. In decision making, other theories concerning epistemic value, dispositions, rational control, and evaluative assessment must be brought to bear along- side the processes described in this article (Asma \& Gabriel, 2019; Brady, 2013; Lebrecht et al., 2012; Zeelenberg \& Pieters, 2006). In this final section, I insert a short illustration of how connections made in this paper help us understand the situatedness of social interaction.

As described above, the neural mechanism for valence appears to be dopaminergic activity, which joins midbrain structures with prefrontal networks that code quantitative prediction of expected reward as derived from past experience (Behrens et al., 2009). The syncretic links provided in this article allow us to understand neural processes according to ecological function. For example, in nonsocial contexts, midbrain dopaminergic systems seem to calculate valuerelated predictions, including reward value (Chang et al., 2013). This process is an essential element in the general process of reinforcement learning wherein neurons in the PFC monitor the value of rewards received by oneself or others, whereas midbrain dopaminergic neurons integrate this information in a feedback loop to generate subjective value (dal Monte et al., 2018). To bring this network into its social setting: in primates, like humans and macaques, who live in large, hierarchical groups that include cohabitation and reciprocity, value associated with social information is signaled by the OFC and ventromedial PFC (Chang, 2017). The social information in primates is, in fact, agent specific and encoded in the PFC; the lateral PFC encodes actions and the dorsomedial PFC encodes task-relevant correct and incorrect actions of 
partner monkeys (dal Monte et al., 2018). The relational nature of the dorsomedial PFC (along with part of the posterior cingulate and a region of the anterior cingulate) is like- wise illustrated in its role in joint attention, theory of mind, and various forms of social cognition (Chang et al., 2013). Building on the discussion of conventions and cultural affordances above, research suggests that depending on the task, variables involved in social processing are grounded in usespecific frames of reference, either centered on the self (egocentric) or situated in the environment (allocentric; Chang, 2017). Social variables thus appear to function in several areas involved in motivation and affect by way of "agent-specific reference frames, contextual gain modulations and. . privileged information processing, and mirroring" (Chang, 2017, p. 1). The task-specific goals innervated by broader motivational thrust blend with the valence generated by the perceptual information to give the perceived object a value that affords decision making in a situated context. Affect-as-motivation in this scenario thus has a role in navigation, contexts of learning, general social skills, and task-relevant social interactions.

\section{Conclusion}

This paper integrates elements of ecological psychology and affective neuroscience to suggest a model for the function of affect-as-motivation. Affect is conceptualized as a conative embodied signal. The felt property of salience within the perceptual world occurs via affective goads that dynamically covary with homeokinetic and exogenous needs, leading to adaptive action patterns and information-seeking behaviors. Affect motivates perception as the internal context that locks onto relevant invariant aspects of the environment to shape subpersonal processes, which then serve the intentional aspects of act-planning (Gallagher, 2009; Hufendiek, 2017; Pacherie, 2011). The unity of perception-action systems qua affordances are depicted as learned vehicles of imperative informational transfer between the creature and the learned context of the social and spatial environment (Heft, 2007; Hutto \& Myin, 2012). This is illustrated by empirical work on salience in attentional processing in the prefrontal lobe. The bias signal of affect-as-motivation is an intention embodied in direct perception. This was illustrated through neuroscientific data on action selection via affective goads dynamically motivated by homeostatic needs that lead to adaptive action patterns and information-seeking behaviors. This work was brought to bear upon the nature of the relation between emotion, cognition, and attention.

\section{Funding}

The author received no financial support for the research, authorship, and/or publication of this article.

\section{ORCID iD}

Rami Gabriel https://orcid.org/0000-0001-5102-9231

\section{Notes}

1. Navigation refers to both spatial and social navigation (see Schafer and Schiller, 2018).

2. For example, Berridge et al. (2009) on pleasure circuits, as well as autopoiesis as metabolism in Thompson (2004). 
3. The term goad is similar to incentives but has phenomenal connotations. It is not as general as drives; see Panksepp (1998) on the Law of Affect.

4. This approach is in contrast to social psychology, which places its focus on higher level attitude objects as mental constructs or schemas (see Fazio, 1995; Gabriel, 2007; GinerSorolla, 1999), or attitudinal theories that describe the action readiness of emotional evaluative states (Deonna \& Teroni, 2012; Frijda, 2007; Prinz, 2004).

5. Language realizes values by creating, pausing, and modifying intention and will (Hodges, 2015).

6. I do not rule out a role for representation in symbol manipulation (Asma \& Gabriel, 2019).

7. To do so would be to put forward a continuation of the computational theory of mind. My goal is rather to offer an alternative in which perception is direct and affect as salience mediates action selection in the mindbrain.

8. Other areas implicated in a "reward circuit" are: the subthalamic nuclei (located at the inter-face between basal ganglia and limbic cortex), the dorsal and ventral hippocampal formation, the extended amygdala, the ventral pallidum, the lateral habenula, and the rostromedial tegmental nucleus (McGinty et al., 2011).

\section{References}

Alcaro, A., \& Panksepp, J. (2011). The SEEKING mind: Primal neuro-affective substrates for appetitive incentive states and their pathological dynamics in addictions and depression. Neuroscience \& Biobehavioral Reviews, 35(9), $1805-1820$. https://doi.org/10.1016/j.neubi- orev.2011.03.002

Ashenfelter, K. T., Boker, S. M., Waddell, J. R., \& Vitanov, N. (2009). Spatiotemporal sym- metry and multifractal structure of head movements during dyadic conversation. Journal of Experimental Psychology: Human Perception and Performance, 35(4), 1072-1091. https:// doi.org/10.1037/a0015017

Asma, S. T., \& Gabriel, R. (2019). The emotional mind: The affective roots of culture and cognition. Harvard University Press.

Azzi, J. C. B., Sirigu, A., \& Duhamel, J.- R. (2012). Modulation of value representation by social context in the primate orbitofrontal cortex. Proceedings of the National Academy of Sciences of the United States of America, 109(6), $2126-2131$. https://doi.org/10.1073/pnas.1111715109

Badyaev, A. V., \& Uller, T. (2009). Parental effects in ecology and evolution: Mechanisms, processes and implications. Philosophical Transactions of the Royal Society B: Biological Sciences, 364(1520), 1169-1177. https://doi.org/10.1098/rstb.2008.0302

Bar-On, D. (2017). Communicative intentions, expressive communication, and origins of mean- ing. In K. Andrews \& J. Beck (Eds.), Routledge companion to the philosophy of animal minds (pp. 301-312). Routledge.

Bechara, A., Damasio, A. R., Damasio, H., \& Anderson, S. W. (1994). Insensitivity to future con- sequences following damage to human prefrontal cortex. Cognition, 50(1-3), 7-15. https:// doi.org/10.1016/0010-0277(94)90018-3

Behrens, T. E., Hunt, L. T., \& Rushworth, M. F. (2009). The computation of social behavior. Science, 324(5931), $1160-1164$. https://doi.org/10.1126/science.1169694

Berridge, K. C., \& Kringelbach, M. L. (2013). Neuroscience of affect: Brain mechanisms of pleasure and displeasure. Current Opinion in Neurobiology, 23(3), 294-303. https://doi. org/10.1016/j.conb.2013.01.017

Berridge, K. C., Robinson, T. E., \& Aldridge, J. W. (2009). Dissecting components of reward: "Liking," "wanting," and "learning." Current Opinion in Pharmacology, 9(1), 65-73. https:// doi.org/10.1016/j.coph.2008.12.014 
Brady, M. S. (2013). Emotional insight: The epistemic role of emotional experience. Oxford University Press.

Cacioppo, S., Bolmont, M., \& Monteleone, G. (2018). Spatio-temporal dynamics of the mirror neuron system during social intentions. Social Neuroscience, 13(6), 718-738. https://doi.org /10.1080/17470919.2017.1394911

Cashdan, E. (1998). Smiles, speech, and body posture: How women and men display socio- metric status and power. Journal of Nonverbal Behavior, 22(4), 209-228. https://doi. org/10.1023/A:1022967721884

Chang, S. W. C. (2017, September 29). An emerging field of primate social neurophysiology: Current developments. eNeuro, 4(5). https://doi.org/10.1523/ENEURO.0295-17.2017

Chang, S. W. C., Brent, L. J. N., Adams, G. K., Klein, J. T., Pearson, J. M., Watson, K. K., \& Platt, M. L. (2013). Neuroethology of primate social behavior. Proceedings of the National Academy of Sciences of the United States of America, 110(Supp. 2), 10387-10394. https:// doi.org/10.1073/pnas.1301213110

Chemero, A. (2009). Radical embodied cognitive science. MIT Press.

Cisek, P. (2007). Cortical mechanisms of action selection: The affordance competition hypothesis. Philosophical Transactions of the Royal Society B: Biological Sciences, 362(1485), 1585- 1599. https://doi.org/10.1098/rstb.2007.2054

Cisek, P., \& Kalaska, J. F. (2010). Neural mechanisms for interacting with a world full of action choices. Annual Review of Neuroscience, 33, 269-298. https://doi.org/10.1146/annurev. neuro.051508.135409

Cole, P. (2006). Cultural variations in the socialization of young children's anger and shame. Child Development, 77, $1237-1251$. https://doi.org/10.2307/3878429

Cole, P., Zahn-Waxler, C., Fox, N. A., Usher, B. A., \& Welsh, J. D. (1996). Individual differences in emotion regulation and behavior problems in preschool children. Journal of Abnormal Psychology, 105, 518-529. https://doi.org/10.1037/0021843X.105.4.518

Colombetti, G., \& Krueger, J. (2015). Scaffoldings of the affective mind. Philosophical Psychology, 28(8), 1157-1176. https://doi.org/10.1080/09515089.2014.976334

Crapse, T. B., Lau, H., \& Basso, M. A. (2018). A role for the superior colliculus in decision crite- ria. Neuron, 97(1), $181-194$. https://doi.org/10.1016/j.neuron.2017.12.006

dal Monte, O., Fan, S., \& Chang, S. W. C. (2018). Social subjective value in the primate midbrain. Nature Neuroscience, 21, 1298-1299. https://doi.org/10.1038/s41593-018-0230-1

Damasio, A. R. (1994). Descartes' error: Emotion, reason, and the human brain. Putnam. Damasio, A. R. (1996). The somatic marker hypothesis and the possible functions of the prefrontal cortex. Philosophical Transactions of the Royal Society B: Biological Sciences, 351(1346), 1413-1420. https://doi.org/10.1098/rstb.1996.0125

Damasio, A. R. (2018). The strange order of things: Life, feeling, and the making of cultures. Pantheon Press.

Davidson, R. J., Scherer, K. R., \& Goldsmith, H. (2009). Handbook of affective sciences. Oxford University Press. 
de Haan, S., Rietveld, E., Stokhof, M., \& Denys, D. (2013). The phenomenology of deep brain stimulation-induced changes in OCD: An enactive affordance-based model. Frontiers in Human Neuroscience, 7, Article 653.

https://doi.org/10.3389/fnhum.2013.00653

de Jaegher, H., \& di Paolo, E. (2007): Participatory sense-making. An enactive approach to social cognition. Phenomenology and the Cognitive Sciences, 6, 485-507. https://doi.org/10.1007/ s11097-007-9076-9

Deonna, J., \& Teroni, F. (2012). The emotions: A philosophical introduction. Routledge. Desimone, R., \& Duncan, J. (1995). Neural mechanisms of selective visual attention. Annual Review of Neuroscience, 18, 193-222.

https://doi.org/10.1146/annurev.ne.18.030195.001205 de Waal, F. (Ed.). (2001). Tree of origin: What primate behavior can tell us about human social evolution. Harvard University Press.

Dickinson, A., \& Balleine, B. (1994). Motivation control of goal-directed action. Animal Learning and Behavior, 22, 1-18. https://doi.org/10.3758/BF03199951

Dorris, M. C., \& Glimcher, P. W. (2004). Activity in posterior parietal cortex is correlated with the relative subjective desirability of action. Neuron, 44(2), 365-378. https://doi.org/10.1016/j. neuron.2004.09.009

Dovidio, J. F., Brown, C. E., Heltman, K., Ellyson, S. L., \& Keating, C. F. (1988). Power displays between women and men in discussions of gender-linked tasks: A multichannel study. Journal of Personality and Social Psychology, 55(4), $580-587$. https://doi.org/10.1037/0022- 3514.55.4.580

Drake, J. M. (2003). What has ecology to do with psychology? Theory \& Psychology, 13(4), 573-576. https://doi.org/10.1177/09593543030134008

Dreyfus, H., \& Kelly, S. D. (2007). Heterophenomenology: Heavy-handed sleight-of-hand. Phenomenology and the Cognitive Sciences 6(1-2), 45-55. https://doi.org/10.1007/s11097-006-9042-y

Ellis, S. (1997). Strategy choice in sociocultural context. Developmental Review, 17(4), 490-524. https://doi.org/10.1006/drev.1997.0444

Ellyson, S. L., Dovidio, J. F., Corson, R. L., \& Vinicur, D. L. (1980). Visual dominance behav- ior in female dyads: Situational and personality factors. Social Psychology Quarterly, 43(3), 328-336. https://doi.org/10.2307/3033735

Fazio, R. H. (1995). Attitudes as object-evaluation association: Determinants, consequences, and correlates of attitude accessibility. In R. E. Petty \& J. A. Krosnick (Eds.), Attitude strength: Antecedents and consequences (pp. 247-282). Lawrence Erlbaum.

Fibiger, H. C., \& Phillips, A. G. (1986). Reward, motivation, cognition: Psychobiology of mesote- lencephalic dopamine systems. In F. E. Bloom \& S. D. Geiger (Eds.), Handbook of physiol- ogy: The nervous system (Vol. 4, pp. 647-675). American Physiology Society.

Fiebich, A. (2014). Perceiving affordances and social cognition. In M. Gallotti \& J. Michael (Eds.), Perspectives in social ontology and social cognition, (Vol. 4, pp. 149-166). Springer.

Figee, M., Luigjes, J., Smolders, R., Valencia-Alfonso, C.-E., van Wingen, G., de Kwaasteniet, B., Mantione, M., Ooms, P., de Koning, P., Vulink, N., Levar, N., Droge, L., van den Munckhof, P., Schuurman, P. R., Nederveen, A., van den Brink, W., Mazaheri, A., Vink, M., \& Denys, D. (2013). Deep brain stimulation restores frontostriatal network activity in obsessive-compulsive disorder. Nature Neuroscience, 16, 386-387. https://doi.org/10.1038/nn.3344 
Fischer, A. H., \& Manstead, A. (2015). Social functions of emotion and emotion regulation. In M. Lewis, J. Haviland-Jones, \& L. F. Barrett (Eds.), Handbook of emotions (4th ed., pp. 456-470). Guilford.

Fodor, J. A. (1998). Concepts: Where cognitive science went wrong. Oxford University Press. Fodor, J. A., \& Pylyshyn, Z. W. (1981). How direct is visual perception? Some reflections on Gibson's "ecological approach." Cognition, 9(2), $139-196$. https://doi.org/10.1016/0010-0277(81)90009-3

Frijda, N. H. (1986). The emotions. Cambridge University Press.

Frijda, N. H. (2007). The laws of emotion. Lawrence Erlbaum Associates.

Gabriel, R. (2007). Affective reactions in a prosopagnosic patient (UMI No.: 3274419) [Doctoral dissertation, University of California, Santa Barbara]. ProQuest Dissertations and Theses Global.

Gabriel, R. (2012). Modularity in cognitive psychology and affective neuroscience (pp. 19-25). In J. Panksepp et al., The philosophical implications of affective neuroscience. Journal of Consciousness Studies, 19(3-4), 6-48. https://www.psychologytoday.com/sites/default/files/ attachments/109303/jcs-articlefinal.pdf

Gallagher, S. (2009). Philosophical antecendents to situated cognition. In P. Robbins \& M. Aydede (Eds.), The Cambridge handbook of situated cognition (pp. 35-51). Cambridge University Press.

Gallagher, M., McMahan, R. W., \& Schoenbaum, G. (1999). Orbitofrontal cortex and representa- tion of incentive value in associative learning. The Journal of Neuroscience, 19(15), 6610- 6614. https://doi.org/10.1523/JNEUROSCI.19-15-06610.1999

Gibson, J. J. (1967). New reasons for realism. Synthese, 17(1), 162-172. https://doi.org/10.1007/ BF00485025

Gibson, J. J. (1979). The ecological approach to visual perception. Houghton Mifflin. Giner-Sorolla, R. (1999). Affect in attitude: Immediate and deliberative perspectives. In S. Chaiken \& Y. Trope (Eds.), Dual-process theories in social psychology (pp. 441-461). Guilford Press. Golonka, S. (2015). Laws and conventions in language-related behaviors. Ecological Psychology, 27(3), 236-250. https://doi.org/10.1080/10407413.2015.1068654

Gottlieb, J. P., Kusunoki, M., \& Goldberg, M. E. (1998, January 29). The representation of visual salience in monkey parietal cortex. Nature, 391, 481-484. https://doi.org/10.1038/35135 Graziano, M. S. A. (2016). Ethological action maps: A paradigm shift for the motor cortex. Trends in Cognitive Sciences, 20(2), 121-132. https://doi.org/10.1016/j.tics.2015.10.008

Griffiths, P., \& Scarantino, A. (2009). Emotions in the wild: The situated perspective on emotion. In P. Robbins \& M. Aydede (Eds.), The Cambridge handbook of situated cognition (pp. 437-453). Cambridge University Press.

Harlé, K. M., Allen, J. J. B., \& Sanfey, A. G. (2010). The impact of depression on social economic decision making. Journal of Abnormal Psychology, 119(2), 440-446. https://doi.org/10.1037/ a0018612

Heft, H. (2007). The social constitution of perceiver-environment reciprocity. Ecological Psychology, 19(2), 85-105. https://www.tandfonline.com/doi/abs/10.1080/10407410701331934

Heft, H. (2010). Affordances and the perception of landscape: An inquiry into environmental perception and aesthetics. In C. W. Thompson, P. Aspinall, \& S. Bell (Eds.), Innovative approaches to researching landscape and health (pp. 9-32). Routledge. 
Heras-Escribano, M., \& de Pinedo-García, M. (2018). Affordances and landscapes: Overcoming the nature-culture dichotomy through niche construction theory. Frontiers in Psychology, 8, Article 2294. https://doi.org/10.3389/fpsyg.2017.02294

Hodges, B. H. (2015). Language as a values-realizing activity: Caring, acting, and perceiving. Zygon: Journal of Religion \& Science, 50(3), 711-735. https://doi.org/10.1111/zygo.12196

Hufendiek, R. (2017). Affordances and the normativity of emotions. Synthese, 194, 4455-4476. https://doi.org/10.1007/s11229016-1144-7

Hufendiek, R. (2018). Explaining embodied emotions-With and without representations. Philosophical Explorations, 21(2), 319-331. https://doi.org/10.1080/13869795.2018.147 7985

Hutto, D. D., \& Myin, E. (2012). Radicalizing enactivism: Basic minds without content. MIT Press.

Iberall, A. S. (1995). A physical (homeokinetic) foundation for the Gibsonian theory of perception and action. Ecological Psychology, 7(1), 37-68. https://doi.org/10.1207/s15326969eco0701_3 Iberall, A. S., \& McCulloch, W. S. (1969). The organizing principle of complex living systems. Journal of Basic Engineering, 91(2), 290-294. https://doi.org/10.1115/1.3571099

Ju, W., \& Takayama, L. (2009). Approachability: How people interpret automatic door move- ment as gesture. International Journal of Design, 3(2). http://www.ijdesign.org/index.php/ IJDesign/article/viewFile/574/244

Kahneman, D., \& Tversky, A. (Eds.). (2000). Choices, values and frames. Cambridge University Press.

Kono, T. (2009). Social affordances and the possibility of ecological linguistics. Integrative Psychological \& Behavioral Science, 43, Article 356. https://doi.org/10.1007/s12124-009- 9097-8

Krueger, J., \& Szanto, T. (2016). Extended emotions. Philosophy Compass, 11(12), 863-878. https://doi.org/10.1111/phc3.12390

Kusunoki, M., Gottlieb, J., \& Goldberg, M. E. (2000). The lateral intraparietal area as a salience map: The representation of abrupt onset, stimulus motion, and task relevance. Vision Research, 40(10-12), 1459-1468. https://doi.org/10.1016/S00426989(99)00212-6

Laland, K., Odling-Smee, J., \& Endler, J. (2017). Niche construction, sources of selection and trait coevolution. Interface Focus, 7(5), Article 20160147. https://doi.org/10.1098/rsfs.2016.0147 Laland, K., Odling-Smee, J., \& Myles, S. (2010). How culture shaped the human genome: Bringing genetics and the human sciences together. Nature Reviews Genetics, 11, 137-148. https://doi.org/10.1038/nrg2734

Laland, K. N., Uller, T., Feldman, M. W., Sterelny, K., Müller, G. B., Moczek, A., Jablonka, E., \& Odling-Smee, J. (2015). The extended evolutionary synthesis: Its structure, assumptions and predictions. Proceedings of the Royal Society B: Biological Sciences, 282(1813), Article 20151019. https://doi.org/10.1098/rspb.2015.1019

Lebrecht, S., Bar, M., Barrett, L. F., \& Tarr, M. J. (2012). Micro-valences: Perceiving affective valence in everyday objects. Frontiers in Psychology, 3, Article 107. https://doi.org/10.3389/ fpsyg.2012.00107

Lewontin, R. (1984). Not in our genes: Biology, ideology, and human nature. Pantheon Books. Lipton, P. A., Alvarez, P., \& Eichenbaum, H. (1999). Crossmodal associative memory representa- tions in rodent orbitofrontal cortex. Neuron, 22(2), 349359. https://doi.org/10.1016/s0896- 6273(00)81095-8 
Lo Presti, P. (2020). Persons and affordances. Ecological Psychology, 32(1), 25-40. https://doi.org $/ 10.1080 / 10407413.2019 .1689821$

Manstead, A. S. R., \& Fischer, A. H. (2001). Social appraisal: The social world as object of and influence on appraisal processes. In K. R. Scherer, A. Schorr, \& T. Johnstone (Eds.), Appraisal processes in emotion: Theory, methods, research (pp. 221-232). Oxford University Press.

Marsh, K. (2015). Affordances and interpersonal coordination. In P. Passos, J. Y. Chow, \& K. Davids (Eds.), Interpersonal coordination and performance in social systems (pp. 300-314). Routledge.

McCall, C., Blascovich, J., Young, A., \& Persky, S. (2009). Proxemic behaviors as predictors of aggression towards Black (but not White) males in an immersive virtual environment. Social Influence, 4(2), 138-154. https://doi.org/10.1080/15534510802517418

McCall, C., \& Singer, T. (2015). Facing off with unfair others: Introducing proxemic imaging as an implicit measure of approach and avoidance during social interaction. PLOS ONE, 10(2), Article e0117532. https://doi.org/10.1371/journal.pone.0117532

McGinty, V. B., Hayden, B. Y., Heilbronner, S. R., Dumont, E. C., Graves, S. M., Mirrione, M. M., du Hoffmann, J., Sartor, G. C., España, R. A., Millan, E. Z., Difeliceantonio, A. G., Marchant, N. J., Napier, T. C., Root, D. H., Borgland, S. L., Treadway, M. T., Floresco, S. B., McGinty, J. F., \& Haber, S. (2011). Emerging, reemerging, and forgotten brain areas of the reward circuit: Notes from the 2010 Motivational Neural Networks conference. Behavioural Brain Research, 225(1), 348-357. https://doi.org/10.1016/j.bbr.2011.07.036

McGinty, V. B., Rangel, A., \& Newsome, W. T. (2016). Orbitofrontal cortex value signals depend on fixation location during free viewing. Neuron, 90(6), 1299-1311. https://doi.org/10.1016/j. neuron.2016.04.045

Michaels, C. F., Withagen, R., Jacobs, D. M., Zaal, F. T. J. M., \& Bongers, R. M. (2001). Information, perception, and action: A reply to commentators. Ecological Psychology, 13(3), 227-244. https://doi.org/10.1207/S15326969ECO1303_3

Mignault, A., \& Chaudhuri, A. (2003). The many faces of a neutral face: Head tilt and percep- tion of dominance and emotion. Journal of Nonverbal Behavior, 27(2), 111-132. https://doi. org/10.1023/A:1023914509763

Millikan, R. G. (1996). Pushmi-Pullyu representations. In J. E. Tomberlin (Ed.), Philosophical perspectives: Vol. 9. AI, connectionism, and philosophical psychology (pp. 185-200). Ridgeview.

Narvaez, D., Panksepp, J., Schore, A. N., \& Gleason, T. R. (Eds.). (2012). Evolution, early expe- rience and human development: From research to practice and policy. Oxford University Press.

Neuberg, S. L., Kenrick, D. T., \& Schaller, M. (2010). Evolutionary social psychology. In S. T. Fiske, D. T. Gilbert, \& G. Lindzey (Eds.), Handbook of social psychology (pp. 761-796). John Wiley \& Sons.

https://doi.org/10.1002/9780470561119.socpsy002021

Norman, D. A. (2002). Emotion and design: Attractive things work better. Interactions Magazine, ix(4), 36-42.

https://jnd.org/emotion_design_attractive_things_work_better/

Okon-Singer, H., Hendler, T., Pessoa, L., \& Shackman, A. J. (2015). The neurobiology of emotion- cognition interactions: Fundamental questions and strategies for future research. Frontiers in Human Neuroscience, 9, Article 58.

https://doi.org/10.3389/fnhum.2015.00058

Pacherie, E. (2011). Nonconceptual representations for action and the limits of intentional control. Social Psychology, 42(1), 6773. https://doi.org/10.1027/1864-9335/a000044

Padmala, S., Bauer, A., \& Pessoa, L. (2011). Negative emotion impairs conflict-driven executive control. Frontiers in Psychology, 2, Article 192. https://doi.org/10.3389/fpsyg.2011.00192

Panksepp, J. (1998). Affective neuroscience: The foundations of human and animal emotions. Oxford University Press. 
Panksepp, J., \& Biven, L. (2012). The archaeology of mind: Neuroevolutionary origins of human emotions. W. W. Noton.

Pears, A., Parkinson, J. A., Hopewell, L., Everitt, B. J., \& Roberts, A. C. (2003). Lesions of the orbitofrontal but not medial prefrontal cortex disrupt conditioned reinforcement in primates. The Journal of Neuroscience, 23(35), 11189-11201.

https://doi.org/10.1523/ JNEUROSCI.23-35-11189.2003

Pessoa, L. (2013). The cognitive-emotional brain. MIT Press.

Pessoa, L. (2018). Emotion and the interactive brain: Insights from comparative neuroanatomy and complex systems. Emotion Review, 10(3), 204-216. https://doi.org/10.1177/175 4073918765675

Pezzulo, G. (2011). Grounding procedural and declarative knowledge in sensorimotor anticipation. Mind \& Language, 26(1), 78114. https://doi.org/10.1111/j.1468-0017.2010.01411.x Pezzulo, G., \& Cisek, P. (2016). Navigating the affordance landscape: Feedback control as a pro- cess model of behavior and cognition. Trends in Cognitive Science, 20(6), 414-424. https:// doi.org/10.1016/j.tics.2016.03.013

Phelps, E. (2006). Emotion and cognition: Insights from studies of the human amygdala. Annual Review of Psychology, 57, 2753. https://doi.org/10.1146/annurev.psych.56.091103.070234

Platt, M., \& Padoa-Schioppa, C. (2009). Neuronal representations of value. In P. W. Glimcher, C. F. Camerer, E. Fehr, \& R. A. Poldrack (Eds.), Neuroeconomics: Decision making and the brain (pp. 441-462). Elsevier Academic Press.

Pollock, J. L. (2001). Evaluative cognition. Nous, 35(3), 325-364. https://doi.org/10.2307/2671891 Prinz, J. (2004). Gut reactions: A perceptual theory of emotion. Oxford University Press.

Ramstead, M. J. D., Veissière, S. P. L., \& Kirmayer, L. J. (2016). Cultural affordances: Scaffolding local worlds through shared intentionality and regimes of attention. Frontiers in Psychology, 7, Article 1090. https://doi.org/10.3389/fpsyg.2016.01090

Reed, E. S. (1986). James J. Gibson's revolution in perceptual psychology: A case study of the transformation of scientific ideas. Studies in the History and Philosophy of Science: Part A, 17(1), 65-98. https://doi.org/10.1016/0039-3681(86)90019-1

Reed, E. S. (1996). Encountering the world: Toward an ecological psychology. Oxford University Press.

Rietveld, E. (2012). Bodily intentionality and social affordances in context. In F. Paglieri (Ed.), Consciousness in interaction: The role of the natural and social context in shaping conscious- ness (pp. 207-226). John Benjamins.

https://doi.org/10.1075/aicr.86.11rie

Rietveld, E., de Haan, S., \& Denys, D. (2013). Social affordances in context: What is it that we are bodily responsive to? Behavioral and Brain Sciences 36(4), 436. https://doi.org/10.1017/ S0140525X12002038

Rietveld, E., \& Kiverstein, J. (2014). A rich landscape of affordances. Ecological Psychology, 26(4), 325-352. https://doi.org/10.1080/10407413.2014.958035

Robinson, S., \& Pallasmaa, J. (Eds.). (2017). Mind in architecture: Neuroscience, embodiment, and the future of design. MIT Press.

Rogoff, B. (2003). The cultural nature of human development. Oxford University Press.

Rolls, E. T., Hornak, J., Wade, D., \& McGrath, J. (1994). Emotion-related learning in patients with social and emotional changes associated with frontal lobe damage. Journal of Neurology, Neurosurgery \& Psychiatry, 57(12), 1518-1524.

https://doi.org/10.1136/jnnp.57.12.1518

Runeson, S. (1988). The distorted room illusion, equivalent configurations, and the specificity of static optic arrays. Journal of Experimental Psychology: Human Perception and Performance, 14(2), 295-304. https://doi.org/10.1037/0096-1523.14.2.295 
Runeson, S., \& Frykholm, G. (1983). Kinematic specification of dynamics as an informational basis for person-and-action perception: Expectation, gender recognition, and deceptive intention. Journal of Experimental Psychology: General, 112(4), 585-615. https://doi. org/10.1037/0096-3445.112.4.585

Schafer, M., \& Schiller, D. (2018). Navigating social space. Neuron, 100(2), 476-489.

https://doi.org/10.1016/i.neuron.2018.10.006

Schoenbaum, G., Chiba, A. A., \& Gallagher, M. (1998). Orbitofrontal cortex and basolateral amygdala encode expected outcomes during learning. Nature Neuroscience, 1, 155-159. https://doi.org/10.1038/407

Schultz, W., Tremblay, L., \& Hollerman, J. R. (2000). Reward processing in primate orbitofrontal cortex and basal ganglia. Cerebral Cortex, 10(3), 272-283. https://doi.org/10.1093/cer-cor/10.3.272

Shackman, A. J., Salomons, T. V., Slagter, H. A., Fox, A. S., Winter, J. J., \& Davidson, R. J. (2011, February 18). The integration of negative affect, pain and cognitive control in the cingulate cortex. Nature Reviews Neuroscience, $12,154-167$. https://doi.org/10.1038/nrn2994

Shaw, R. (2003). The agent-environment interface: Simon's indirect or Gibson's direct coupling? Ecological Psychology, 15(1), 37-106. https://doi.org/10.1207/S15326969ECO1501 04

Shaw, R., \& Kinsella-Shaw, J. (2007). The survival value of informed awareness. Journal of Consciousness Studies, 14(1-2), 137-154. http://commons.trincoll.edu/robertshaw/ files/2016/02/RES_JKS_2007.pdf

Spinoza, B. (2001). Ethics (W. H. White, Trans.). Wordsworth Editions. (Original work published 1677)

Sterelny, K. (2012). The evolved apprentice: How evolution made humans unique. MIT Press.

Thompson, E. (2004). Life and mind: From autopoiesis to neurophenomenology. A tribute to Francisco Varela. Phenomenology and the Cognitive Sciences, 3, 381-398. https://doi.org/10.1023/B:PHEN.0000048936.73339.dd

Tomasello, M. (2000). Culture and cognitive development. Current Directions in Psychological Science, 9(2), 37-40. https://doi.org/10.1111/1467-8721.00056

Turvey, M. T. (1992). Affordances and prospective control: An outline of the ontology. Ecological Psychology, 4(3), $173-187$. https://doi.org/10.1207/s15326969eco0403_3

Turvey, M. T., Shaw, R. E., Reed, E. S., \& Mace, W. M. (1981). Ecological laws of perceiving and acting: In reply to Fodor and Pylyshyn (1981). Cognition, 9(3), 237-304. https://doi. org/10.1016/0010-0277(81)90002-0

Van Acker, R., \& Valenti, S. S. (1989). Perception of social affordances by children with mild handicapping conditions: Implications for social skills research and training. Ecological Psychology, 1(4), 383-405.

https://doi.org/10.1207/s15326969eco0104_3

Wallis, J. D. (2007). Orbitofrontal cortex and its contribution to decision-making. Annual Review of Neuroscience, 30, 31-56. https://doi.org/10.1146/annurev.neuro.30.051606.094334

Warren, W. H. (1988). Action modes and laws of control for the visual guidance of action. In O. Meijer \& K. Roth (Eds.), Movement behavior: The motor-action controversy (pp. 339-380). North Holland. 
Warren, W. H. (2006). The dynamics of perception and action. Psychological Review, 113(2), 358-389.

https://doi.org/10.1037/0033-295X.113.2.358

Watt, D. F. (2017). Reflections on the neuroscientific legacy of Jaak Panksepp (1943-2017). Neuropsychoanalysis, 19(2), 183198. https://doi.org/10.1080/15294145.2017.1376549

Wilson, M., \& Wilson, T. P. (2005). An oscillator model of the timing of turn-taking. Psychonomic Bulletin \& Review, 12, 957968. https://doi.org/10.3758/BF03206432

Wise, R. A., \& Rompré, P.-P. (1989). Brain dopamine and reward. Annual Review of Psychology, 40, $191-225$.

https://doi.org/10.1146/annurev.ps.40.020189.001203

Withagen, R., \& Chemero, A. (2009). Naturalizing perception: Developing the Gibsonian approach to perception along evolutionary lines. Theory \& Psychology, 19(3), 363-389. https://doi. org/10.1177/0959354309104159

Withagen, R., \& Michaels, C. (2005). On ecological conceptualizations of perceptual systems and action systems. Theory \& Psychology, 15(5), 603-620. https://doi.org/10.1177/0959 354305057265

Zeelenberg, M., \& Pieters, R. (2006). Feeling is for doing: A pragmatic approach to the study of emotions in economic behavior. In D. de Cremer, M. Zeelenberg, \& J. K. Murnighan (Eds.), Social psychology and economics (pp. 117-137). Lawrence Erlbaum Associates.

Zipoli Caiani, S., \& Ferretti, G. (2017). Semantic and pragmatic integration in vision for action. Consciousness and Cognition, 48, 40-54. https://doi.org/10.1016/j.concog.2016.10.009

\section{Author biography}

Rami Gabriel received his doctorate in cognitive and perceptual sciences in 2007 under Dr. Stanley B. Klein for a dissertation on prosopagnosia and the relation between emotion and cognition. His main research interests are philosophy of psychology, affective neuroscience, consciousness stud- ies, self, and identity. Recent publications include (with S. T. Asma), The Emotional Mind: Affective Roots of Culture and Cognition (Harvard University Press, 2019) and Why I Buy: Self, Taste, and Consumer Society in America (Intellect Press, 2011). 